\title{
On the Relative Merits of Three-Point Integration Rules
}

\author{
for Six-Node Triangles
}

\author{
K.Y.Sze and D.Zhu* \\ Department of Mechanical Engineering, The University of Hong Kong \\ Pokfulam Road, HONG KONG
}

\begin{abstract}
There exist two three-point integration rules for triangular elements. Both rules are precise up to the second order and used for evaluating the six-node triangles. While one of rules has its sampling stations inside the triangle, that of the other coincide with the edge nodes. Though the former is commonly employed, it will be seen in this short paper that latter is indeed more favourable in view of element accuracy.
\end{abstract}

Keywords : triangle, numerical integration, three-point, plate bending, membrane, finite element

* on leave from Southwest Jiaotong University, Chengdu, Sichuan 610031, P.R.CHINA

Published in Finite Elements in Analysis and Design 27 (1997) 335-343 


\section{Quadratic Triangles}

Fig. 1 shows a six-node triangle. Cartesian coordinates $(x, y)$, displacement components $(u, v, w)$ and rotations about the Cartesian coordinate axes $\left(\theta_{x}, \theta_{y}\right)$ are interpolated in terms of the area coordinates $(r, s)$ :

$$
\begin{aligned}
& x=\sum_{i=1}^{6} N_{i} x_{i}, y=\sum_{i=1}^{6} N_{i} y_{i} \\
& u=\sum_{i=1}^{6} N_{i} u_{i}, v=\sum_{i=1}^{6} N_{i} v_{i}, w=\sum_{i=1}^{6} N_{i} w_{i}, \theta_{x}=\sum_{i=1}^{6} N_{i} \theta_{x i}, \theta_{y}=\sum_{i=1}^{6} N_{i} \theta_{y i}
\end{aligned}
$$

where

$$
\begin{aligned}
& N_{1}=(1-s-t)(1-2 s-2 t), N_{2}=s(2 s-1), N_{3}=t(2 t-1), \\
& N_{4}=4 s t, N_{5}=4 t(1-s-t), N_{6}=4(1-s-t) s
\end{aligned}
$$

The set of interpolation functions $N_{i}$ 's is complete up to the second order of $s$ and $t$. From Eqn.(1), Jacobian matrix, its determinant and inverse can be worked out :

$$
\mathbf{J}=\left[\begin{array}{ll}
\partial_{s} x & \partial_{s} y \\
\partial_{t} x & \partial_{t} y
\end{array}\right], J=\operatorname{det} .(\mathbf{J})=\left(\partial_{s} x\right)\left(\partial_{t} y\right)-\left(\partial_{t} x\right)\left(\partial_{s} y\right) \quad \text { and } \quad \mathbf{J}^{-1}=\frac{1}{J}\left[\begin{array}{cc}
\partial_{t} y & -\partial_{s} y \\
-\partial_{t} x & \partial_{s} x
\end{array}\right]
$$

in which $\partial_{s}=\frac{\partial}{\partial s}$ and $\partial_{t}=\frac{\partial}{\partial t}$. Moreover,

$$
\partial_{x}=\frac{\partial}{\partial x}=\frac{\partial_{t} y}{J} \partial_{s}-\frac{\partial_{s} y}{J} \partial_{t} \text { and } \partial_{y}=\frac{\partial}{\partial y}=-\frac{\partial_{t} x}{J} \partial_{s}+\frac{\partial_{s} x}{J} \partial_{t}
$$

When the element is subparametric (all the edge nodes are at the middle of the corresponding corner nodes), Cartesian coordinates are linear functions of the area coordinates and vice versa, i.e.

$$
x=(1-s-t) x_{1}+s x_{2}+t x_{3}, y=(1-s-t) y_{1}+s y_{2}+t y_{3}
$$

Thus, the interpolations given in Eqn.(1) and Eqn.(2) are also complete up to the second order of $x$ and $y$. Moreover,

$$
\begin{aligned}
& J=\left(x_{2}-x_{1}\right)\left(y_{3}-y_{1}\right)-\left(y_{2}-y_{1}\right)\left(x_{3}-x_{1}\right)=2 A \\
& \partial_{x}=\frac{\left(y_{3}-y_{1}\right)}{2 A} \partial_{s}-\frac{\left(y_{2}-y_{1}\right)}{2 A} \partial_{t} \text { and } \partial_{y}=-\frac{\left(x_{3}-x_{1}\right)}{2 A} \partial_{s}+\frac{\left(x_{2}-x_{1}\right)}{2 A} \partial_{t}
\end{aligned}
$$

where $A$ denotes the area of the subparametric triangle. 
For the six-node membrane triangle, the element membrane strain components are derived from the interpolated displacement components, namely :

$$
\left\{\begin{array}{l}
\varepsilon_{x} \\
\varepsilon_{y} \\
\gamma_{x y}
\end{array}\right\}=\left[\begin{array}{cc}
\partial_{x} & 0 \\
0 & \partial_{y} \\
\partial_{y} & \partial_{x}
\end{array}\right]\left\{\begin{array}{l}
u \\
v
\end{array}\right\}=\left[\begin{array}{cc}
\partial_{x} & 0 \\
0 & \partial_{y} \\
\partial_{y} & \partial_{x}
\end{array}\right] \mathbf{N}_{m} \mathbf{q}_{m}=\mathbf{B}_{m} \mathbf{q}_{m}
$$

where

$$
\mathbf{N}_{m}=\left[N_{1} \mathbf{I}_{2}, N_{2} \mathbf{I}_{2}, \ldots, N_{5} \mathbf{I}_{2}, N_{6} \mathbf{I}_{2}\right], \mathbf{q}_{m}=\left[u_{1}, v_{1}, \ldots, u_{6}, v_{6}\right]^{T}
$$

and $\mathbf{I}_{\mathrm{n}}$ denotes the $\mathrm{n}$-th order identity matrix. The element stiffness of the membrane element, $K_{m}$, is obtained by computing the membrane strain energy inside the element, i.e.

$$
\mathbf{K}_{m}=\left\langle\mathbf{B}_{m}^{T} \mathbf{C}_{m} \mathbf{B}_{m} J\right\rangle
$$

in which $\mathbf{C}_{m}$ is the inplane material stiffness matrix and $\langle f(s, t)\rangle=\int_{0}^{1} \int_{0}^{1-t} f(s, t) d s d t$ is the integral operator over the element area in its $s$-t-plane as portrayed in the right hand side of Fig.1.

For $\mathrm{C}^{0}$ plate bending elements [1-3], the displacement components at any point $(x, y, z)$ inside the element are governed by :

$$
u=u(x, y, z)=z \theta_{y}(x, y), v=v(x, y, z)=-z \theta_{x}(x, y), w=w(x, y)
$$

The element strain components of the six-node triangle are obtained from the interpolated deflection and rotations :

$$
\begin{gathered}
\left\{\begin{array}{c}
\varepsilon_{x} \\
\varepsilon_{y} \\
\gamma_{x y}
\end{array}\right\}=z\left[\begin{array}{ccc}
0 & 0 & \partial_{x} \\
0 & -\partial_{y} & 0 \\
0 & -\partial_{x} & \partial_{y}
\end{array}\right]\left\{\begin{array}{c}
w \\
\theta_{x} \\
\theta_{y}
\end{array}\right\}=z\left[\begin{array}{ccc}
0 & 0 & \partial_{x} \\
0 & -\partial_{y} & 0 \\
0 & -\partial_{x} & \partial_{y}
\end{array}\right] \mathbf{N}_{p} \mathbf{q}_{p}=z \mathbf{B}_{b} \mathbf{q}_{p} \\
\left\{\begin{array}{l}
\gamma_{z x} \\
\gamma_{z y}
\end{array}\right\}=\left[\begin{array}{ccc}
\partial_{x} & 0 & 1 \\
\partial_{y} & -1 & 0
\end{array}\right]\left\{\begin{array}{l}
w \\
\theta_{x} \\
\theta_{y}
\end{array}\right\}=\left[\begin{array}{ccc}
\partial_{x} & 0 & 1 \\
\partial_{y} & -1 & 0
\end{array}\right] \mathbf{N}_{p} \mathbf{q}_{p}=\mathbf{B}_{s} \mathbf{q}_{p}, \quad \varepsilon_{z}=\partial_{z} w=0
\end{gathered}
$$

where

$$
\mathbf{N}_{p}=\left[N_{1} \mathbf{I}_{3}, N_{2} \mathbf{I}_{3}, \ldots, N_{5} \mathbf{I}_{3}, N_{6} \mathbf{I}_{3}\right], \mathbf{q}_{p}=\left[w_{1}, \theta_{x 1}, \theta_{y 1}, \ldots, w_{6}, \theta_{x 6}, \theta_{y 6}\right]^{T}
$$

After integrating the bending and transverse shear strain energy with respect to $z$ from $-h / 2$ to $+h / 2$ where $h$ is the plate thickness, the stiffness matrix of the plate bending element is obtained as : 


$$
\mathbf{K}_{P}=\frac{h^{3}}{12}\left\langle\mathbf{B}_{b}^{T} \mathbf{C}_{b} \mathbf{B}_{b} J\right\rangle+h\left\langle\mathbf{B}_{s}^{T} \mathbf{C}_{s} \mathbf{B}_{s} J\right\rangle
$$

where $\mathbf{C}_{b}$ and $\mathbf{C}_{s}$ are the material stiffness matrices for the inplane plane stress and transverse shear responses, respectively. The first and second terms in the right hand side of the above equation are the bending and transverse shear stiffness matrices, respectively.

Some properties of the six-node membrane and plate bending triangles which are evaluated by three-point integration rules are listed in Table 1 . It can be seen that the difference between the number of nodal d.o.f.s and the number of rigid body modes equals to the total number of sampled strain components. Numerical experiments also reveal that none of the elements possesses spurious zero energy mode. Hence, three-point rules are just sufficient to secure the element rank sufficiency. As the accuracy of displacement-based elements often deteriorates with the excessive number of integration stations (that is the reason why analysts prefer using reduced integration unless the spurious zero energy modes cannot be suppressed by the prescribed boundary conditions), both rules may be considered as optimal for the six-node triangles.

Table 1. Some properties of the quadratic membrane and plate bending triangles

\begin{tabular}{|c|c|c|}
\hline & membrane triangle & plate bending triangle \\
\hline no. of nodal d.o.f.s - no. of rigid body modes & $6 \times 2-3=9$ & $6 \times 3-3=15$ \\
\hline no. of strain components & 3 & 5 \\
\hline total no. of sampled strain components & $3 \times 3=9$ & $3 \times 5=15$ \\
\hline no. of spurious zero energy modes & nil & nil \\
\hline
\end{tabular}

\section{Three-Point Integration Rules}

The area coordinates $(r, s)$ and weighting factors of the sampling stations for the two three-points integration rules are :

$$
\begin{array}{ll}
\text { Rule I : }\left(\frac{1}{6}, \frac{1}{6}\right),\left(\frac{1}{6}, \frac{2}{3}\right),\left(\frac{2}{3}, \frac{1}{6}\right) & ; \text { weighting factors }=\frac{1}{6} \\
\text { Rule II : }\left(\frac{1}{2}, \frac{1}{2}\right),\left(0, \frac{1}{2}\right),\left(\frac{1}{2}, 0\right) & ; \text { weighting factors }=\frac{1}{6}
\end{array}
$$

It can seen that the sampling stations of Rule I are well inside the element whereas the stations of Rule II coincide with the edge nodes. The integral operators based on Rule I and Rule II can respectively be expressed as :

$$
\langle f(s, t)\rangle_{I}=\frac{1}{6}\left(f\left(\frac{1}{6}, \frac{1}{6}\right)+f\left(\frac{1}{6}, \frac{2}{3}\right)+f\left(\frac{2}{3}, \frac{1}{6}\right)\right)
$$

and 


$$
\langle f(s, t)\rangle_{I I}=\frac{1}{6}\left(f\left(\frac{1}{2}, \frac{1}{2}\right)+f\left(\frac{1}{2}, 0\right)+f\left(0, \frac{1}{2}\right)\right)
$$

By applying the two rules to the membrane element $\mathbf{K}_{m}$ and plate bending element $\mathbf{K}_{p}$, we have

$$
\begin{aligned}
\mathbf{K}_{m-I} & =\left\langle\mathbf{B}_{m}^{T} \mathbf{C}_{m} \mathbf{B}_{m} J\right\rangle_{I}, \mathbf{K}_{m-I I}=\left\langle\mathbf{B}_{m}^{T} \mathbf{C}_{m} \mathbf{B}_{m} J\right\rangle_{I I} \\
\mathbf{K}_{P-I} & =\frac{h^{3}}{12}\left\langle\mathbf{B}_{b}^{T} \mathbf{C}_{b} \mathbf{B}_{b} J\right\rangle_{I}+h\left\langle\mathbf{B}_{s}^{T} \mathbf{C}_{s} \mathbf{B}_{s} J\right\rangle_{I}, \mathbf{K}_{P-I I}=\frac{h^{3}}{12}\left\langle\mathbf{B}_{b}^{T} \mathbf{C}_{b} \mathbf{B}_{b} J\right\rangle_{I I}+h\left\langle\mathbf{B}_{s}^{T} \mathbf{C}_{s} \mathbf{B}_{s} J\right\rangle_{I I}
\end{aligned}
$$

Most of the texts [1-4] written by finite element researchers mention both three-point rules but none of them comments on the relative merits of the two rules. On the other hand, Rule I appears to be far more popular than Rule II. In the following sections, their relative merits are studied.

\section{MacNeal's Cantilever Problem for Quadratic Membrane Triangles}

In this section, $\mathbf{K}_{m-I}$ and $\mathbf{K}_{m-I I}$ are contrasted by MacNeal's slender cantilever problem [4]. The cantilever is of dimension $1 \times 30$ and loaded by an end shear force P as shown in Fig.2a. At the supported end, all nodal d.o.f.s are restrained. The twelve membrane triangles are arranged in pairs that form trapezoids. Results for two slightly different meshes are computed. Besides the subparametric mesh, a non-subparametric mesh is formed by shifting four of the edge nodes horizontally to the centres of the corresponding trapezoids, see Fig.2b. After being normalized by the thin beam solution, Table 2 lists the end deflections of $\mathbf{K}_{m-I}$ and $\mathbf{K}_{m-I I}$ as well as the ones given in MacNeal's text [4] in which the Poisson's ratio is not specified.

Table 2. Normalized tip deflection for the slender cantilever problem, see Fig.2

\begin{tabular}{|c|c|c|c|c|}
\hline \multirow{2}{*}{$\begin{array}{c}\text { six-node } \\
\text { element }\end{array}$} & \multicolumn{2}{|c|}{ subparametric mesh } & \multicolumn{2}{c|}{ non-subparametric mesh } \\
\cline { 2 - 5 } & Poisson's ratio $=0$ & $\begin{array}{c}\text { Poisson's ratio }= \\
0.3\end{array}$ & Poisson's ratio $=0$ & $\begin{array}{c}\text { Poisson's ratio }= \\
0.3\end{array}$ \\
\hline $\mathbf{K}_{m-I}$ & 0.973 & 0.962 & 0.468 & 0.460 \\
$\mathbf{K}_{m-I I}$ & 0.973 & 0.962 & 0.820 & 0.820 \\
\hline MacNeal & \multicolumn{2}{|c|}{0.953 (unknown Poisson's ratio) } & \multicolumn{2}{c|}{0.391 (unknown Poisson's ratio) } \\
\hline
\end{tabular}

For subparametric membrane triangles, the integrand $\left(\mathbf{B}_{m}^{T} \mathbf{C}_{m} \mathbf{B}_{m} J\right)$ is second order in $s$ and $t$. Since Rule I and Rule II are both precise up to the same order, subparametric $\mathbf{K}_{m-I}$ and $\mathbf{K}_{m-I I}$ are identical. However, $\mathbf{K}_{m-I I}$ is much more accurate than $\mathbf{K}_{m-I}$ when they are not subparametric. Though MacNeal's results are slightly different from that of $\mathbf{K}_{m-I}$, it is believed that Rule I is employed in his tests. The minor difference may be due to the discrepancies in setting the boundary conditions, mesh geometry and material constants. 
To elaborate the poor performance of six-node membrane element in the non-subparametric mesh, MacNeal considered the element illustrated in Fig.3. Origin of the coordinates is at the midpoint of nodes 2 and 3 . Thus, the nodal coordinates are :

$$
\begin{aligned}
& x_{1}=-\Lambda, \quad x_{2}=\Lambda, \quad x_{3}=-\Lambda, \quad x_{4}=\alpha \Lambda, \quad x_{5}=-\Lambda, \quad x_{6}=0 \\
& y_{1}=-1, \quad y_{2}=-1, \quad y_{3}=1, \quad y_{4}=0, y_{5}=0, \quad y_{6}=-1
\end{aligned}
$$

After invoking the nodal interpolation functions,

$$
\left\{\begin{array}{l}
x \\
y
\end{array}\right\}=\left\{\begin{array}{c}
-\Lambda+2 \Lambda s+4 \alpha \Lambda s t \\
2 t-1
\end{array}\right\}, \mathbf{J}=4\left[\begin{array}{cc}
\Lambda(1+2 \alpha t) & 0 \\
2 \alpha \Lambda s & 1
\end{array}\right], J=\operatorname{det}(\mathbf{J})=4 \Lambda(1+2 \alpha t)
$$

Nodal displacements of the element is then prescribed according to

$$
\bar{u}=x y, \bar{v}=-x^{2} / 2
$$

which correspond to a pure inplane bending field for zero Poisson's ratio. Thus, the error of the element strain components are :

$$
\begin{aligned}
& E\left(\varepsilon_{x}\right)=\frac{\partial u}{\partial x}-\frac{\partial \bar{u}}{\partial x}=\frac{2 \alpha t(1-2 t)}{1+2 \alpha t}, E\left(\varepsilon_{y}\right)=\frac{\partial v}{\partial y}-\frac{\partial \bar{v}}{\partial y}=\frac{\alpha \Lambda^{2} s(4 s-2-\alpha)}{1+2 \alpha t} \\
& E\left(\gamma_{x y}\right)=\left(\frac{\partial u}{\partial y}+\frac{\partial v}{\partial x}\right)-\left(\frac{\partial \bar{u}}{\partial y}+\frac{\partial \bar{v}}{\partial x}\right)=\frac{\alpha \Lambda(2 s-2 t-\alpha t)}{1+2 \alpha t}
\end{aligned}
$$

where $u$ and $v$, as given in Eqn.(2), are obtained by interpolation. When $\alpha$ is small compared to unity,

$$
E\left(\varepsilon_{x}\right) \cong 2 \alpha t(1-2 t), E\left(\varepsilon_{y}\right) \cong 2 \alpha \Lambda^{2} s(2 s-1), E\left(\gamma_{x y}\right) \cong 2 \alpha \Lambda(s-t)
$$

MacNeal explained the poor performance of the triangle by pointing out the highly detrimental effect of $E\left(\varepsilon_{y}\right)$ for large $\Lambda$.

To see why $\mathbf{K}_{m-I I}$ yields more accurate results than $\mathbf{K}_{m-I}$. Table 3 shows the strain errors computed at the sampling stations of the two integration rules. The higher accuracy of $\mathbf{K}_{m-I I}$ becomes apparent as the errors vanish at most of the sampling stations of Rule II. 
Table 3. Errors of the strain components at the sampling stations of Rule I and Rule II

\begin{tabular}{|c|ccc|ccc|}
\hline & \multicolumn{3}{|c|}{$\mathbf{K}_{m-I}$} & \multicolumn{3}{c|}{$\mathbf{K}_{m-I I}$} \\
\hline integration stations & $(1 / 6,1 / 6)$ & $(1 / 6,2 / 3)$ & $(2 / 3,1 / 6)$ & $(1 / 2,1 / 2)$ & $(0,1 / 2)$ & $(1 / 2,0)$ \\
\hline$E\left(\varepsilon_{x}\right) \cong 2 \alpha t(1-2 t)$ & $2 \alpha / 9$ & $-4 \alpha / 9$ & $2 \alpha / 9$ & 0 & 0 & 0 \\
$E\left(\varepsilon_{y}\right) \cong 2 \alpha \Lambda^{2} s(2 s-1)$ & $-2 \alpha \Lambda^{2} / 9$ & $-2 \alpha \Lambda^{2} / 9$ & $4 \alpha \Lambda^{2} / 9$ & 0 & 0 & 0 \\
$E\left(\gamma_{x y}\right) \cong 2 \alpha \Lambda(s-t)$ & 0 & $-\alpha \Lambda$ & $\alpha \Lambda$ & 0 & $-\alpha \Lambda$ & $\alpha \Lambda$ \\
\hline
\end{tabular}

Experienced practitioners always avoid subparametric geometry. Hence, only very few elements at the curved boundary of the plane continuum have to be non-subparametric. In view of the writer, the present cantilever test is most useful to identify whether a six-node membrane triangle is a good candidate for being generalized to curved shell and large displacement analyses. In both types of analyses, curved element edges cannot be avoided.

\section{Shear Locking of Six-Node $C^{0}$ Plate Bending Triangles}

Shear locking is a common deficiency that plagues thin plate and shell analyses. It is caused by the extreme ratio of the bending strain energy to the transverse shear energy, see Eqn.(13). For subparametric elements, it can be checked that $\left(\mathbf{B}_{s}^{T} \mathbf{C}_{s} \mathbf{B}_{s} J\right)$ is quartic. While both Rule I and Rule II can exactly integrate up to the quadratic terms, the higher order terms in $\left(\mathbf{B}_{s}^{T} \mathbf{C}_{s} \mathbf{B}_{s} J\right)$ are evaluated by the two rules with different approximations. Hence, $\mathbf{K}_{p-I}$ and $\mathbf{K}_{p-I I}$ are different even if the mesh is subparametric. This is in contrast with $\mathbf{K}_{m-I}$ and $\mathbf{K}_{m-I I}$.

The simplest method for pre-identifying locking is perhaps the constraint index, CI, count [1-4]. When an element is added into an existing mesh, it brings forward a number of NK independent kinematic d.o.f.s and a number of NC independent transverse shear constraints. For displacementbased elements, every of the transverse shear components at an integration station constitutes a constraint. $\mathrm{CI}$ is defined as :

$$
\mathrm{CI}=\mathrm{NK}-\mathrm{NC}
$$

For CI less than or equal to zero, an element model has a high risk of locking.

Fig. 4 shows the CI count for $\mathbf{K}_{p-I}$ and $\mathbf{K}_{p-I I}$. The short strokes in the figure indicate the locations of the sampled transverse shear strain components. As the sampling stations of Rule I are well inside the element, CI count for $\mathbf{K}_{p-I}$ is straight forward. For the two transverse shear components at any sampling station (edge node) of Rule II, they can always be transformed into the two components which are normal and tangential to the element edge. The latter is an "edge-constraint" in the sense that only the d.o.f.s of the nodes in the same element edge are involved regardless whether the edge is straight or curved. The hatched element edges in the figure are pre-existing in the mesh. Thus, the d.o.f.s of the nodes along and the edge-constraints sampled at the hatched edges do not contribute to $\mathrm{NK}$ and $\mathrm{NC}$, respectively. Hence, there is only one constraint brought forward 
per point "a". Similarly, among the four constraints sampled at point "c" by the two elements, only three of them are independent. Consequently, NC and CI for $\mathbf{K}_{p-I I}$ are 9 and 3, respectively.

Based on the CI count, $\mathbf{K}_{p-I}$ has a high risk of locking whereas $\mathbf{K}_{p-I I}$ is relatively immune. A clamped square plate problem is employed to test the locking behaviour of $\mathbf{K}_{p-I}$ and $\mathbf{K}_{p-I I}$, see Fig.5. The side length and thickness of plate are denoted by $L$ and $h$, respectively. Both partially clamped $\left(w=\theta_{t}=0\right)$ and fully clamped $\left(w=\theta_{x}=\theta_{y}=0\right)$ conditions are considered. Owing to symmetry, only a quadrant of the plate is analyzed. After normalized by the thin plate solution [5], Table 4 lists the central deflections predicted by the two elements for various $L$ to $h$ ratios. Locking is only observed in $\mathbf{K}_{p-I}$.

Table 4. Normalized central deflections for clamped square plate subjected to central point load

\begin{tabular}{|c|c|c|c|c|c|c|c|c|}
\hline \multirow{2}{*}{} & \multicolumn{4}{|c|}{ partially clamped } & \multicolumn{4}{c|}{ fully clamped } \\
\cline { 2 - 9 } & $2 \times 2$ & mesh & \multicolumn{2}{|c|}{$4 \times 4$ mesh } & \multicolumn{2}{c|}{$2 \times 2$ mesh } & \multicolumn{2}{|c|}{$4 \times 4$ mesh } \\
\cline { 2 - 9 } & $\mathbf{K}_{p-I}$ & $\mathbf{K}_{p-I I}$ & $\mathbf{K}_{p-I}$ & $\mathbf{K}_{p-I I}$ & $\mathbf{K}_{p-I}$ & $\mathbf{K}_{p-I I}$ & $\mathbf{K}_{p-I}$ & $\mathbf{K}_{p-I I}$ \\
\hline $10^{2}$ & 0.321 & 0.380 & 0.864 & 0.934 & 0.304 & 0.378 & 0.747 & 0.934 \\
$10^{3}$ & 0.013 & 0.263 & 0.635 & 0.875 & $5.2 \times 10^{-3}$ & 0.261 & 0.355 & 0.875 \\
$10^{4}$ & 0.008 & 0.261 & 0.511 & 0.873 & $5.2 \times 10^{-5}$ & 0.261 & $9.4 \times 10^{-3}$ & 0.873 \\
$10^{5}$ & 0.008 & 0.261 & 0.504 & 0.873 & $5.2 \times 10^{-7}$ & 0.261 & $9.6 \times 10^{-5}$ & 0.873 \\
\hline
\end{tabular}

To further assess the relative accuracy of the two elements, simply supported and partially clamped square plates are analyzed. Under the two boundary conditions, $\mathbf{K}_{p-I}$ are relatively free from locking. $L$ to $h$ ratio of plate is fixed at 1000. After normalized by the thin plate solution, the central deflections are listed in Table 5. It can be seen that $\mathbf{K}_{p-I I}$ is again markedly more accurate than $\mathbf{K}_{p-I}$.

Table 5. Normalized central deflections for simply supported and partially clamped square plate

\begin{tabular}{|cccccc|}
\hline \multirow{2}{*}{ element } & mesh & \multicolumn{2}{c}{ central point load } & \multicolumn{2}{c|}{ uniform pressure } \\
& simply supported & $\begin{array}{l}\text { partially } \\
\text { clamped }\end{array}$ & simply supported & $\begin{array}{c}\text { partially } \\
\text { clamped }\end{array}$ \\
\hline \multirow{3}{*}{$\mathbf{K}_{p-I}$} & $2 \times 2$ & 0.678 & 0.013 & 0.764 & 0.045 \\
& $4 \times 4$ & 0.861 & 0.635 & 0.923 & 0.730 \\
& $8 \times 8$ & 0.958 & 0.915 & 0.982 & 0.960 \\
\hline \multirow{3}{*}{$\mathbf{K}_{p-I I}$} & $2 \times 2$ & 0.844 & 0.263 & 0.939 & 0.509 \\
& $4 \times 4$ & 0.954 & 0.875 & 0.990 & 0.934 \\
& $8 \times 8$ & 0.989 & 0.973 & 1.000 & 0.993 \\
\hline
\end{tabular}

\section{Closure}

Compared to the research on linear triangles and quadratic quadrilaterals, six-node (quadratic) triangles are relatively unexplored. Nevertheless, it is strongly felt that Rule I enjoys a much higher popularity than Rule II. Some indirect "evidence" is as follows. First of all, when the deficiency of 
the six-node membrane triangle are discussed, MacNeal [4] appeared to evaluated the element by Rule I. This point is reflected in the corresponding predictions and has been mentioned in Section 3. Moreover, the writer also analyzed the cantilever problems by the six-node membrane element in ANSYS 5.0a. The element gave the same predictions as $\mathbf{K}_{m-I}$. It can be seen from the constraint index count conducted by Zienkiewicz \& Lefebvre [6] that the referred plate bending triangle is evaluated by Rule I. In the educational paper written by Felippa on how to program the six-node membrane element [7] and in the very recent text of Bathe [8], Rule II is not even mentioned.

This paper aims to point out and explain the fact that Rule II is more favourable than Rule I in view of element accuracy. The finite element community is suggested to give a thought on whether Rule I should still enjoy its present popularity.

Acknowledgment - The financial support of the Research Grants Council of Hong Kong Government is gratefully acknowledged.

\section{References}

[1] T.J.R.Hughes, The Finite Element Method - linear static and dynamic finite element analysis, Prentice-Hall, New Jersey, 1987

[2] O.C.Zienkiewicz, R.L.Taylor, The Finite Element Method, 4 ${ }^{\text {th }}$ Edn., McGraw-Hill, London, 1989

[3] R.D.Cook, D.S.Malkus, Concepts and Applications of Finite Element Analysis, $3^{\text {rd }}$ edn., John Wiley \& Sons, New York, 1989

[4] R.H.MacNeal, Finite Elements : Their Design and Performance, Marcel Dekker, New York, 1994

[5] S.P.Timoshenko, S.Woinowsky-Krieger, Theory of Plates and Shells, 2nd Edn., McGraw-Hill, New York, 1970

[6] O.C.Zienkiewicz, D.Lefebvre, "A robust triangular plate bending element of the ReissnerMindlin type", Inter.J.Numer.Methods Engrg., 26, 1169-1184 (1988)

[7] C.A.Felippa, "Programming the isoparametric six-node triangle", Engineering Computations, 7, 173-177 (1990)

[8] K.-J.Bathe, Finite Element Procedures, Prentice Hall, New Jersey, 1996 

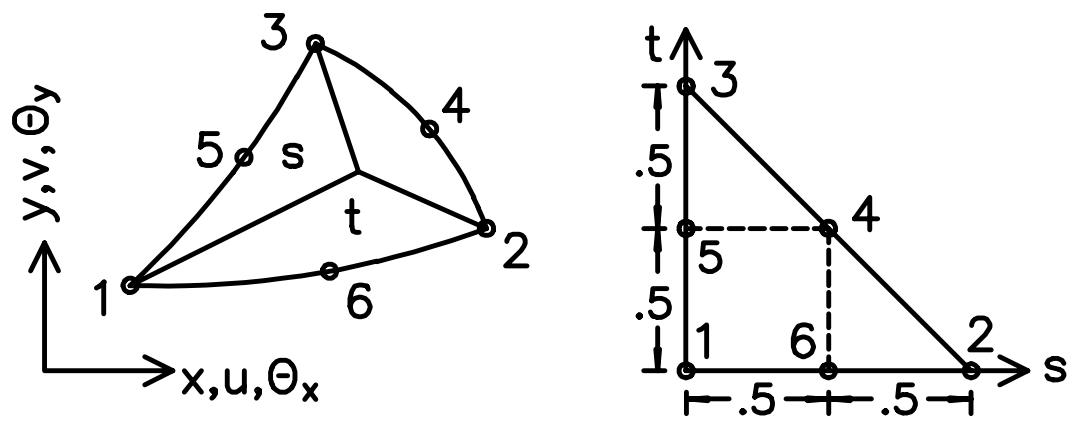

Fig.1 (Left) Six-node triangle in the x-y-plane, (righ) the element in its area s-t-plane

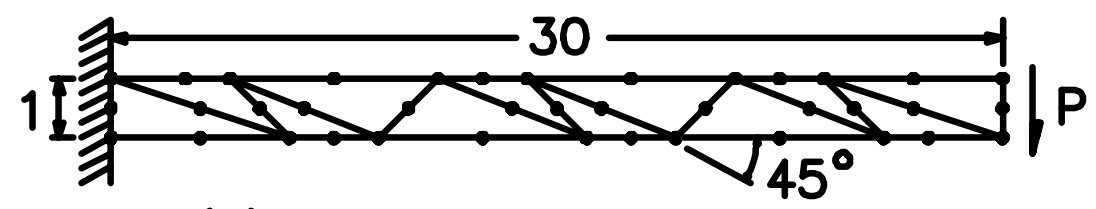

(a) overview of the cantilever

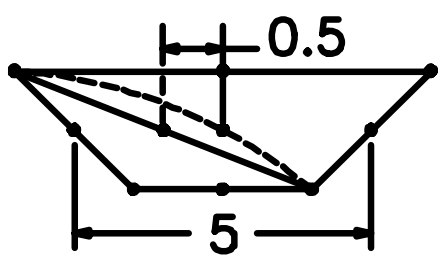

(b) detail of modelling schemes

Fig.2 MacNeal's slender cantilever problem

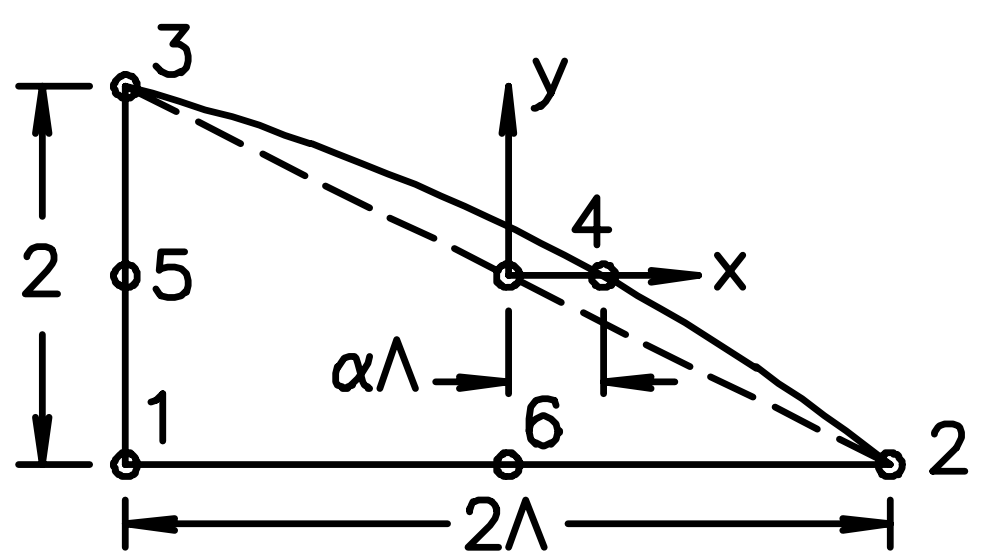

Fig.3 Quadratic triangle with an offset edge node 

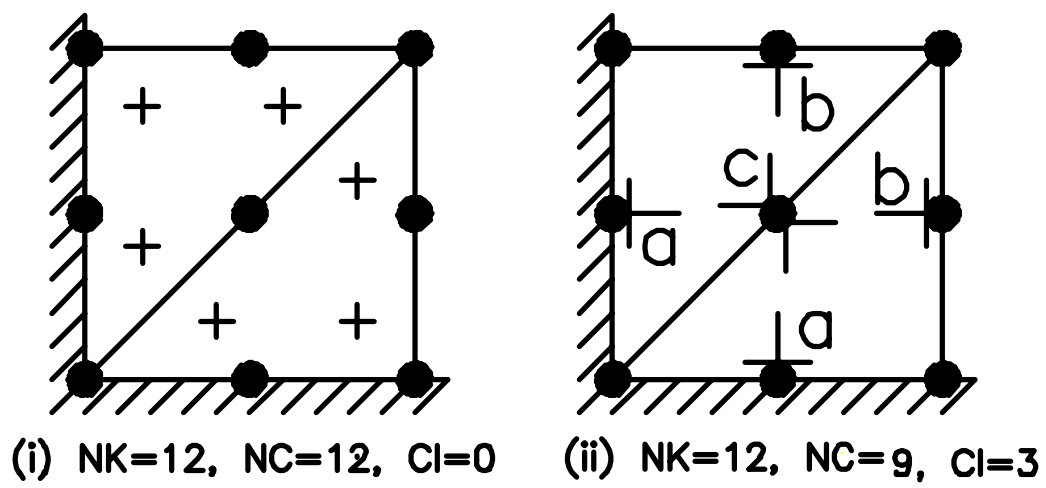

Fig.4 Constraint counts for elements integrated by Rule I (left) and Rule II (right)

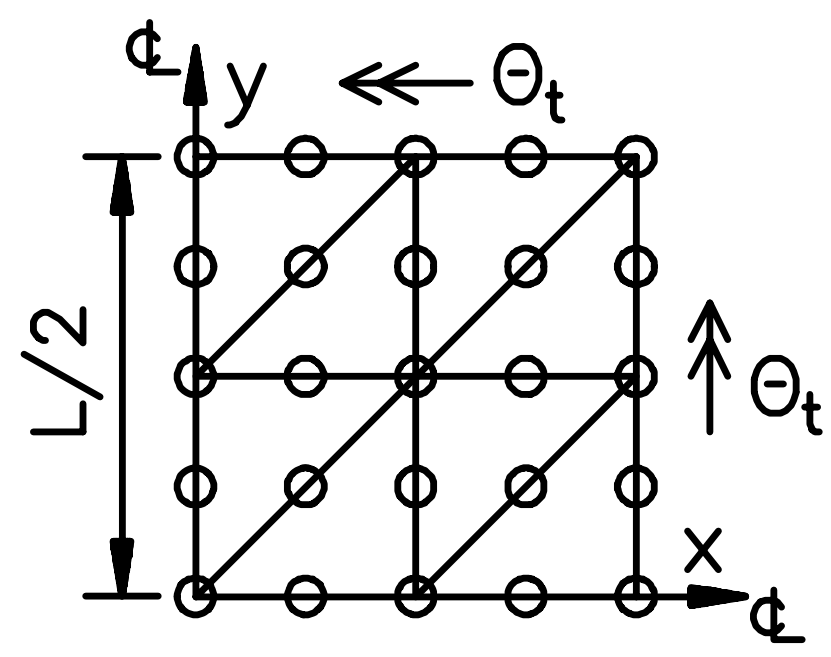

Fig.5 A quadrant of square plate modelling by a $2 \times 2$ mesh ( 8 elements) 